\title{
Ultrafast Kikuchi Diffraction: Nanoscale Stress-Strain Dynamics of Wave-Guiding Structures
}

\author{
Aycan Yurtsever, Sascha Schaefer, and Ahmed H. Zewail* \\ Physical Biology Center for Ultrafast Science and Technology, Arthur Amos Noyes Laboratory of Chemical Physics, California \\ Institute of Technology, Pasadena, California 91125, United States
}

\begin{abstract}
Complex structural dynamics at the nanoscale requires sufficiently small probes to be visualized. In conventional imaging using electron microscopy, the dimension of the probe is large enough to cause averaging over the structures present. However, by converging ultrafast electron bunches, it is possible to select a single nanoscale structure and study the dynamics, either in the image or using electron diffraction. Moreover, the span of incident wave vectors in a convergent beam enables sensitivity levels and information contents beyond those of parallel-beam illumination with a single wave vector Bragg diffraction. Here, we report the observation of propagating strain waves using ultrafast Kikuchi

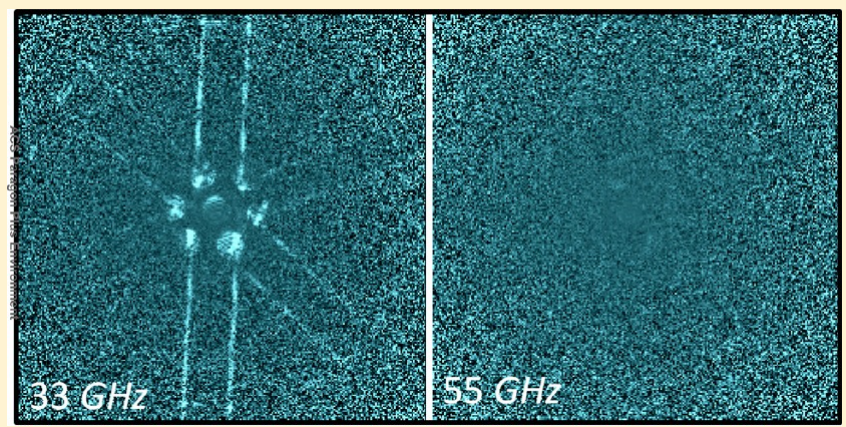
diffraction from nanoscale volumes within a wedge-shaped silicon single crystal. It is found that the heterogeneity of the strain in the lateral direction is only $100 \mathrm{~nm}$. The transient elastic wave gives rise to a coherent oscillation with a period of $30 \mathrm{ps}$ and with an envelope that has a width of 140 ps. The origin of this elastic deformation is theoretically examined using finite element analysis; it is identified as propagating shear waves. The wedge-shaped structure, unlike parallel-plate structure, is the key behind the traveling nature of the waves as its angle permits "transverse" propagation; the parallel-plate structure only exhibits the "longitudinal" motion. The studies reported suggest extension to a range of applications for nanostructures of different shapes and for exploring their ultrafast eigen-modes of stress-strain profiles.
\end{abstract}

KEYWORDS: UEM, convergent electron beams, electron diffraction, elastic waves, wave-guides

Structural dynamics in solids, molecules, and biological systems are often studied using Bragg diffraction in parallel beam illumination. Both electron and X-ray techniques are available for such studies and have demonstrated a wide range of dynamical phenomena that can be captured with ultrafast temporal resolution (for a recent book, see ref 1 ). The use of parallel beams means that the probed areas are on the order of micrometers and that the reciprocal space is probed only by a single incident wave vector. Because of the latter many Bragg peaks, such as the ones of higher Laue zones, are missed. Moreover, with a single wave vector a point on the "continuous" relrods in reciprocal space is probed, and a series of rocking curve measurements is needed. Of relevance here is the fact that important elastic deformations, such as shear motions of the unit cell, are difficult to observe with conventional Bragg diffraction., ${ }^{2,3}$

Convergent ultrafast electron microscopy ${ }^{2-5}$ overcomes these limitations of parallel beam illumination. The electron beam can be focused to a few nanometers, thus allowing ultrafast detection of nonuniform deformations in the lateral direction. Because electrons probe only a small volume of the structure, strong Kikuchi lines are present in the diffraction patterns. $^{6-9}$ These lines are more sensitive to shear deformations than Bragg spots, as we shall discuss below.

In this Letter, we report theoretical analysis and experimental observations of Kikuchi band dynamics, both elucidate the nature of strain deformations. The stress in the material is impulsively induced through a heating ultrafast pulse. As shown below, the wedge geometry with a linear increase in slab thickness produces a propagating shear deformation that is initiated by the laser-driven thermal expansion of the slab and originated at the tip of the wedge. Qualitatively, a compressional expansion in the longitudinal direction results in the socalled breathing modes of the slab. Contrary to the breathing dynamics of a thin sheet, in a wedge this motion is accompanied by transverse motion of the unit cell due to a finite Poisson ration of the material. Because of the thicknesses gradient of the wedge, stress builds up on each side of the unit cell which leads to their buckling and the creation of the propagating shear motion.

Bragg versus Kikuchi Diffraction. In diffraction with convergent electron beams, both Bragg and Kikuchi features are formed simultaneously. Bragg diffraction, which gives rise to spot features in parallel beam illumination, yields diffraction discs in convergent beam probing due to the spread of incident electron wave vectors. Kikuchi features are formed as a result of first diffusive phonon-scattered electrons which then undergo elastic Bragg scatterings. In a sense, Kikuchi diffraction is Bragg

Received: May 1, 2012

Published: June 5, 2012 


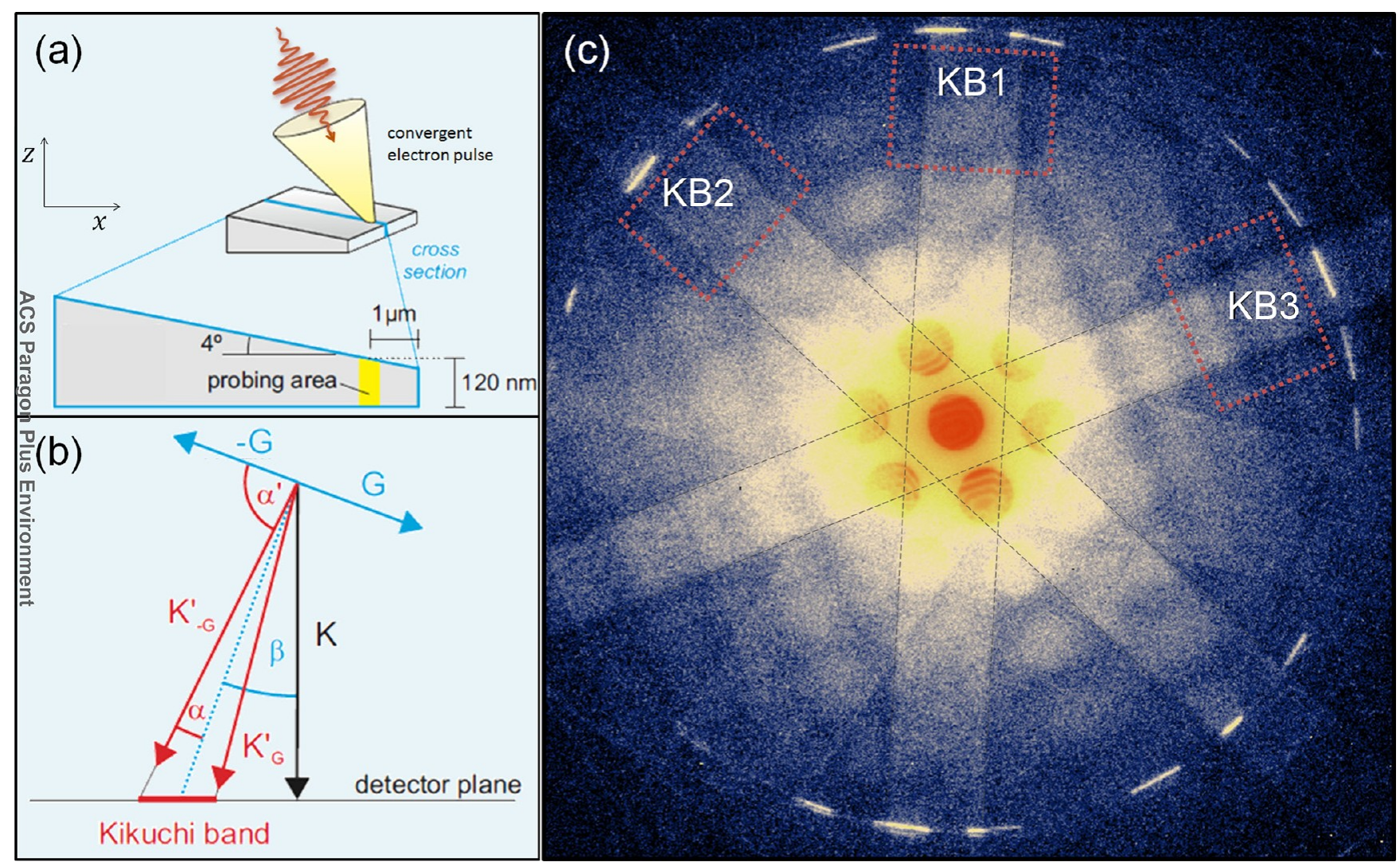

Figure 1. Kikuchi diffraction and geometry of the structure. (a) Geometrical parameters of the silicon wedge specimen. The nanoscale ultrafast electron probe is depicted as a cone. (b) Geometrical construction of the width and location of a Kikuchi band is shown (for details see text). (c) A typical experimental (CBUED) frame taken at negative time, i.e., before the pump and probe pulses overlap in time. Note the bands and discs observed in diffraction, in contrast with Bragg diffraction. The three Kikuchi bands that are the main point of interest in this study are labeled as KB1, $\mathrm{KB} 2$, and $\mathrm{KB} 3$.
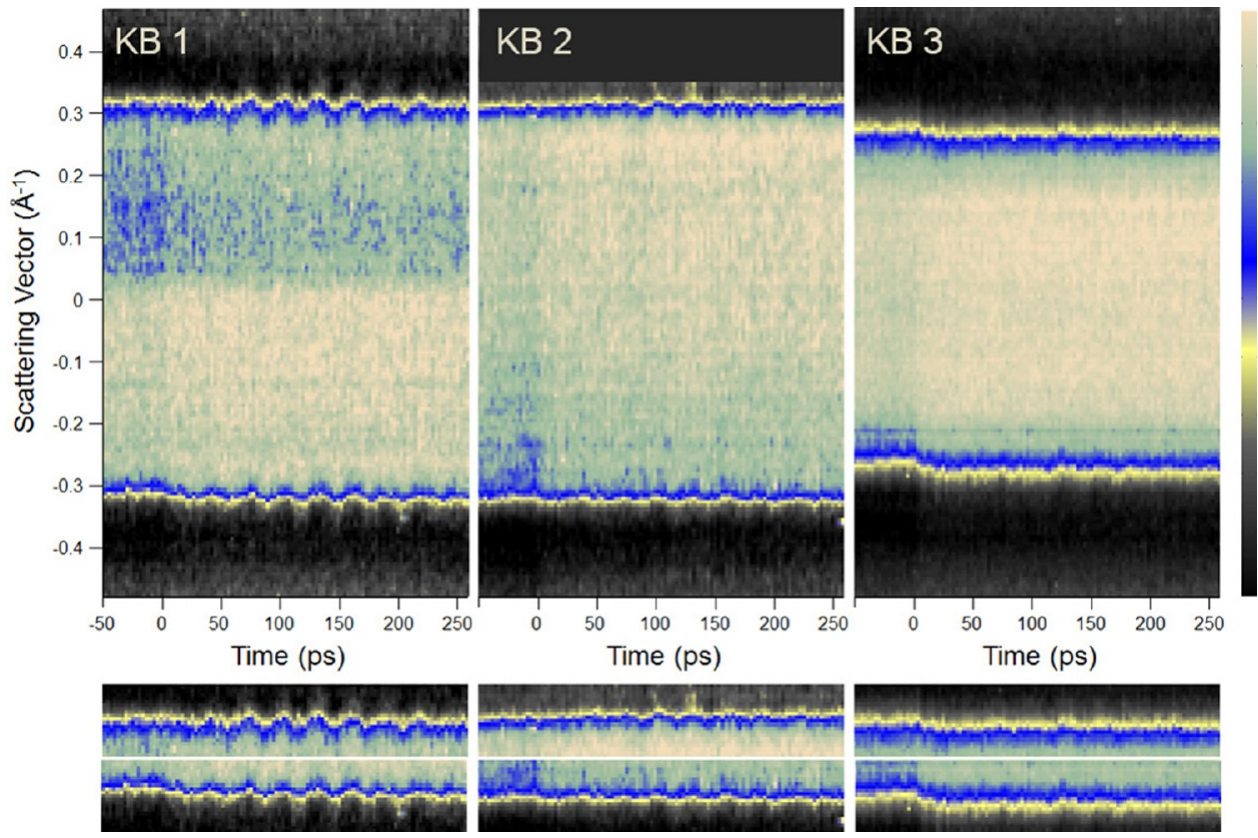

Figure 2. The temporal evolution of the three observed Kikuchi bands. The cross-section of each band is evaluated at the rectangular region indicated in Figure 1c; they are plotted consecutively as a function of the time delay, as shown in the figure. The bottom panel compares the top and bottom edges of the bands in order to reveal the shift and motion in time. The KB1 band shows the strongest oscillation and exhibits a period of 30 ps; KB2 exhibits less modulation. The temporal behavior of the band shift has a "transit-like" nature that peaks at 130 ps. These temporal characteristics, combined with the nanoscale spatial probe, elucidate the nature of heterogeneous structural dynamics, as explained in the text. 


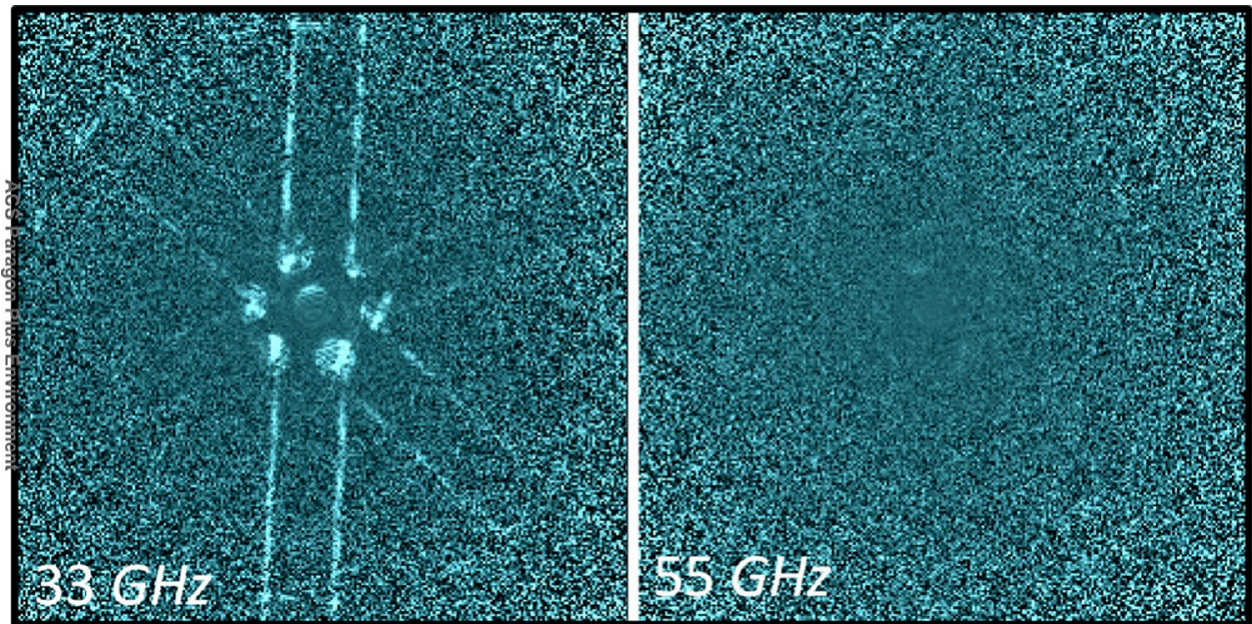

Figure 3. Fourier images, digitally constructed, of the ultrafast dynamics at 33 and $55 \mathrm{GHz}$. While the $33 \mathrm{GHz}$ on-resonance (left) panel clearly exhibits the oscillation at the edges of the Kikuchi bands and inside the CBUED discs, the $55 \mathrm{GHz}$ off-resonance (right) panel does not show any dynamics. As importantly, these data establish that in UEM probing there is enough sensitivity for a signal at each scattering vector (pixel) and that the motion can be temporally analyzed in parallel.

diffraction but with a virtual source located inside the sample and yields a broad range of wave vectors.

Although the information content of the intensity distribution within a convergent-beam-electron-diffraction (CBED) disk is comparable to that of the Kikuchi pattern, we concentrate here on the latter, since the former can only be properly described using multiscattering simulations. In the following, we will restrict our discussion to the position of Bragg spots and Kikuchi lines in terms of their sensitivity and information content, and both can be discussed within the kinematical scattering theory.

There exist important differences between Kikuchi and Bragg diffractions. A change in the Kikuchi band position is a direct projection of the dynamics in reciprocal space, independent of the scattering vector $G$, whereas the change for Bragg spot position is linear in $G$ (Figure 1). In a mathematical form, this change for Kikuchi diffraction, can be expressed as $\Delta \beta=(\Delta a)$ $a) \cdot k_{\text {ino }}$ where $a$ is the unit cell dimension in real space and $\Delta a$ is its change, $k_{\text {inc }}$ is the amplitude of the incident electron wave vector, and $\Delta \beta$ is the change in the position of Kikuchi lines. On the other hand, changes in the position of Bragg reflections are always relative to the length of the lattice vector $(G)$, that is, $\Delta G=(\Delta a / a) \cdot G$. The difference for the two cases reflects the "source" of scattering, as discussed above. Since the incident wave vector $\left(k_{\text {inc }} \cong 40 \AA^{-1}\right)$ is much larger than the reciprocal lattice vectors $\left(G \cong 1 \AA^{-1}\right)$ for a typical solid under the diffraction condition in an electron microscope, Kikuchi lines are 40-100 times more sensitive probes than Bragg reflections. This is the reason why we see in our data 4 pixel oscillations in the Kikuchi bands (Figures 2 and 3), whereas Bragg reflections do not show a significant change.

Results and Discussion. Figure 1 presents the experimental results together with the geometry involved in probing the structure. Figure 1a gives the shape of the structure and the coordinates involved. A wedge shaped Si slab is oriented such that the zone axis of the convergent beam diffraction pattern is [114]. The variables used to discuss the Kikuchi pattern formation are shown in Figure 1b. Figure 1c depicts a typical experimental pattern, in this case that of a single temporal frame of convergent-beam-ultrafast-electron-diffraction (CBUED) scan taken before time zero, that is, before the pump photon and probe electron pulses overlap in time. ${ }^{2}$ The richness of information and sharpness of the features should be noted. Three scans of the same area were performed between -50 and 261 ps with 3 ps steps and with $30 \mathrm{~s}$ acquisition time for each frame. These scans were summed up to optimize the signal-to-noise ratio.

The three main features visible in the diffraction image shown in the figure are the zero-order-Laue-zone (ZOLZ) discs, a higher-order-Laue-zone (HOLZ) ring, and the Kikuchi bands. These bands are labeled as KB1, KB2, and KB3 and are the main point of interest in this paper. Each of these bands corresponds to a different set of crystallographic planes. The $\mathrm{KB} 1$ band is a reflection from the $(1 \overline{3} 1)$ atomic planes, the KB2 from the $(3 \overline{1} 1)$ planes, and KB3 is a reflection from the (220) planes. Imaging the dynamics of such three reflections is sufficient to determine the motion of the unit cell in two dimensions.

The temporal evolution of these three bands is given in Figure 2. At each time delay (or frame) a cross section of the bands was taken in the areas indicated by the dotted red rectangles in Figure 1c. These cross sections were then patched together as a function of time for the three bands, as shown in Figure 2. The most striking dynamics observed in the data is the oscillation of the bands, which is directly visible at the bandedges. The KB1 oscillations are the strongest, in contrast to KB3 which does not show a measurable change; KB2 has weak modulations around 100 ps. These oscillations are in the form of band-shift; the whole Kikuchi band shifts its position while the bandwidth remains the same. This is apparent at the bottom of Figure 2 which compares the opposite edges of each band.

The temporal profile of the shifting motion has an oscillation period of 30 ps. As importantly, these oscillations do not exhibit the expected damping profile of an exponentially decaying function, but rather we observe a "transit-like" dynamics that has a Gaussian-shaped carrier envelope with a full width at halfmaximum of 140 ps and the center at 130 ps. Below, we discuss the origin of this unusual time dependence.

One additional note about the dynamics of Kikuchi bands is that the overall intensity of the bands increases at time-zero as seen in Figure 2. This is a result of ultrafast heating of the $\mathrm{Si}$ 
slab and the subsequent change due to the Debye-Waller effect. As the temperature of the crystal increases the Bragg intensity decreases which has a characteristic decay time of $2 \mathrm{ps}$ for polycrystalline Si measured with parallel beams ${ }^{10}$ and $7 \pm 3$ ps for crystalline $\mathrm{Si}$ measured with nanoscale convergent beams. ${ }^{4}$ These time scales reflect the nature of electronphonon coupling in bulk and finite-sized specimens. ${ }^{11,12}$ Intensity loss in Bragg reflections is compensated with intensity gain between the reflections in the form of thermal diffuse scattering. Hence, the observed intensity increase for the Kikuchi bands.

Although the behavior of the Kikuchi cross sections shown in Figure 2 clearly demonstrates the transient shifting motion, the dynamics in the rest of the pattern are not displayed in the figure. For this purpose, we have developed the following analysis technique. For every given pixel (or scattering vector) in the diffraction pattern, we obtained the Fourier transform of the temporal evolution of their intensity. We marked the amplitude values of this transformation at $33 \mathrm{GHz}$ (i.e., $1 / 30$ ps) and digitally built an image from these values. The results are shown in Figure 3, which clearly shows that the highest intensity is at the edges of KB1. The other bands also depict the expected behavior, following the discussion given above. It is clear that with UEM imaging there is enough signal in the diffraction pattern to analyze each scattering vector simultaneously. For instance, some pixels inside the ZOLZ discs also show the oscillation, which is expected from the tilting motion of the planes. As a consistency check, when the same analysis is done at $55 \mathrm{GHz}$ (Figure 3, right), the band edges do not show any intensity above the noise level.

Diffraction and Eigen Modes of Stress-Strain. A shift in Kikuchi band position is directly related to the tilt of the atomic planes that form the band. These planes are parallel to the direction of probing electrons and the resulting bands are in the ZOLZ. A tilt in atomic planes means that the unit cells along the probing direction experience a buckling deformation. However, the stress and strain involved in the process do not need to be unidirectional.

The relationship between the shift of Kikuchi bands and the elastic strain tensor $(\varepsilon)$ can be quantified. The Laue condition, $2 \boldsymbol{K}^{\prime} \cdot \boldsymbol{G}=G^{2}\left(\boldsymbol{K}^{\prime}\right.$ is the wave vector of the diffracted electron and $G$ is the reciprocal lattice vector), confines $K^{\prime}$ to be lying on a cone around $\boldsymbol{G}$ with an opening angle $\alpha$ given by (see Figure $1 b)$,

$$
\cos \alpha=\sin \alpha^{\prime}=\frac{G}{2 K^{\prime}}
$$

The intersection of this cone with the detector plane generates the Kikuchi line. The angle $\beta$ between the cone and the wave vector $K^{\prime}$ of the incident electron beam, which defines the position of the Kikuchi line in the diffraction pattern (Figure $1 b)$, is given by

$$
\sin \beta \approx \beta=\frac{\mathbf{K}^{\prime} \cdot \mathbf{G}}{K^{\prime} G}
$$

For the Kikuchi lines in Figure 2, $\boldsymbol{K}^{\prime}$ is orthogonal to $\boldsymbol{G}$ so that $\beta=0$. For this case, the area between the Kikuchi lines corresponding to the reciprocal lattice vectors $\boldsymbol{G}$ and $-\boldsymbol{G}$ shows an increased intensity in the diffraction pattern above the background and is called a Kikuchi band, denoted as \pm G These bands arise because of dynamical scattering. The center of the Kikuchi band relative to the incident electron beam is given by the angle $\beta$, and the band has a width of $2 \alpha$. Structural dynamics that lead to a temporal change in the reciprocal lattice have an influence on the width, different from that of its position, of the Kikuchi bands. Using eq 1, the temporal change of the width $\Delta w$ is given by

$$
\frac{\Delta w}{2 \alpha}=\frac{\Delta G}{G}
$$

Taking a typical value of $15 \mathrm{mrad}$ for the width of a Kikuchi band and an excitation-induced structural change of $\Delta G / G \approx$ $10^{-3}$, we obtain a width change of $\Delta w=0.015 \mathrm{mrad}$, which is smaller than the resolution used here.

In contrast, the temporal change of the Kikuchi band position $\beta$ is, in general, non-negligible and can be derived from eq 2 to give

$$
\Delta \beta=\frac{\mathbf{K}^{\prime}}{K^{\prime}} \cdot\left(\frac{\Delta \mathbf{G}}{G}-\frac{\mathbf{G G}^{\mathrm{T}} \cdot \Delta \mathbf{G}}{G^{3}}\right)=\frac{\mathbf{K}^{\prime} \cdot \Delta \mathbf{G}}{K^{\prime} G}=\frac{\mathbf{K}^{\prime} \cdot \varepsilon \mathbf{G}}{K^{\prime} G}
$$

The second term in the parentheses is zero since $\boldsymbol{K}^{\prime} \cdot \boldsymbol{G}=0$ for the cases considered here. Furthermore, we used $\Delta G=\varepsilon \cdot G$ which connects the temporal change of the strain tensor with the observed shift of the Kikuchi band position $\beta$. For the more general case, the complete displacement gradient tensor, $\nabla u$, has to be applied, that is, $\Delta G=\Delta u \cdot G$, instead of only considering the symmetric part, that is, the strain tensor. It is possible to include both the intrinsic shape changes as well as the rotation of the sample. However, in the present case the offdiagonal parts of the displacement gradient tensor are small and can therefore be neglected.

To further elucidate the type of strain, $\varepsilon$, that leads to a change in the Kikuchi band position, we consider the case of isotropic volume expansion $\Delta V / V$. In this case, the strain tensor is diagonal and is given by $\varepsilon=[(\Delta V / V) / 3] \cdot \mathbf{I}$, where $\mathbf{I}$ is the identity matrix. For isotropic dynamics, $\varepsilon \boldsymbol{G}$ has the same direction as $\boldsymbol{G}$ and is normal to $\boldsymbol{K}^{\prime}$, which leads to no change in the Kikuchi band position $(\Delta \beta=0)$. If the relative magnitude of the unilateral expansion is $\lambda$ along a direction $\boldsymbol{n}$ ( $\boldsymbol{n}$ is the normalized direction vector), then the strain tensor is given by $\varepsilon=\lambda \boldsymbol{n}^{\mathrm{T}} \boldsymbol{n}$, where $\boldsymbol{n}^{\mathrm{T}}$ is the transpose of the vector $\boldsymbol{n}$. Using eq 3, we obtain that a change in Kikuchi band position is only observed when the product $\left(\boldsymbol{K}^{\prime} \cdot \boldsymbol{n}\right) \cdot(\boldsymbol{n} \cdot \boldsymbol{G})$ does not vanish. Since $\boldsymbol{K}^{\prime}$ and $\boldsymbol{G}$ are perpendicular to each other, a unilateral expansion in either the direction of $\boldsymbol{K}^{\prime}$ or $\boldsymbol{G}$ does not lead to a shift in the Kikuchi band position whereas a maximal shift is observed when the direction of the expansion is at an angle of $\pi / 4$ to both $K^{\prime}$ and $\boldsymbol{G}$.

Theoretical Simulations. The nature of the heterogeneous structural dynamics and the strain propagation in a wedgeshaped structure can be solved numerically using finite element simulations. In order to simulate the unit cell deformation generated by ultrafast heating, we consider the following set of differential equations

$$
\begin{aligned}
& \rho c_{\mathrm{p}} \frac{\partial T}{\partial t}=\nabla \cdot(\kappa \nabla T)+Q \\
& \rho \frac{\partial^{2} u}{\partial t^{2}}-\nabla \cdot \sigma=0
\end{aligned}
$$

Equation 5 describes the temporal and spatial evolution of the temperature at different points within the sample. Here, $\rho=$ $2330 \mathrm{~kg} / \mathrm{m}^{3}$ is the mass density, $T$ is the local temperature, $c_{\mathrm{p}}=$ $703 \mathrm{~J} /(\mathrm{kg} \cdot \mathrm{K})$ is the specific isobaric heat capacity, and $k=163$ 
$\mathrm{W} /(\mathrm{m} \cdot \mathrm{K})$ is the heat conductivity of silicon. We incorporate the effect of laser excitation in the source term $Q$ with

$$
Q(y, t)=Q_{0} \exp \left(\frac{-y}{\chi}\right) \exp \left(\frac{-t}{\tau}\right)
$$

where we take the penetration depth of the laser beam as $\chi=$ $1100 \mathrm{~nm}, Q_{0}=1.5 \times 10^{20} \mathrm{~W} / \mathrm{m}^{3}$, and a coupling constant of $\tau=$ 2 ps. Smaller changes in $\chi$ and $\tau$ do not significantly affect the simulation results. With this choice of $Q_{0}$, we obtain a steady state temperature rise of $160 \mathrm{~K}$, which is in agreement with our previously reported results. ${ }^{4}$

Equation 6 describes the connection between the spatial change of the stress tensor $\sigma$ and the temporal behavior of the displacement field $u$. Here, $t$ and $\rho$ denote the time coordinate and the density of the material. Since we are considering the effect of a relatively small stress, we can assume that the material behaves elastically so that the stress tensor is given by

$$
\sigma=C:\left[\varepsilon+\alpha\left(T-T_{0}\right)\right]
$$

Here, $C$ is the stiffness tensor, and (:) denotes the double dot product. The thermal expansion tensor that describes the change in the equilibrium structure when the temperature is raised from $T_{0}$ to $T$ is given by $\alpha$.

The strain $\varepsilon$ is connected to the displacement field $u$ by

$$
\varepsilon_{i j}=\frac{1}{2}\left(\frac{\partial u_{i}}{\partial x_{j}}+\frac{\partial u_{j}}{\partial x_{i}}\right)
$$

where $x_{i}(i=x, y, z)$ are the spatial coordinates, and $u_{i}$ is the $i$ th component of the displacement field. With the boundary conditions defined by the structure's wedge geometry, we numerically solved eqs 5-9 using the Comsol 4.2 program suite. The resulting temporal and spatial evolution of the strain field is displayed in Figure 4.

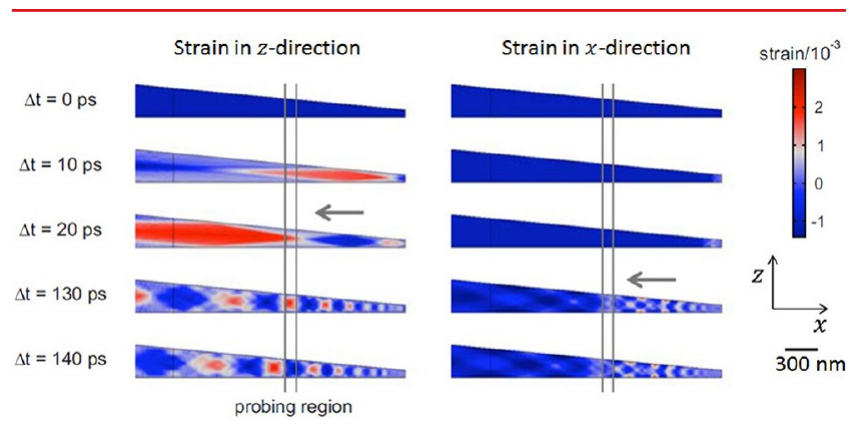

Figure 4. Theoretical simulations of strain dynamics in a wedge shaped structure. The spatial variation of strain along $z$ - (left panel) and $x$-direction (right panel) is illustrated at different delay times after excitation. The probed region is depicted by two vertical lines. Strain originates at the tip of the wedge and propagates as shown (gray arrows). The lateral modulations of the strain are on the order of 100 $\mathrm{nm}$, which necessitates the use of nanoscale probes in order to visualize such dynamics.

In general, the strain field is defined by six components, that is, the three diagonal components of the strain tensor as well as the three off-diagonal shear components. However, in the case considered here, the symmetry of the wedge allows us to constrain the dynamics to within the cutting plane shown in the left panel of Figure 1. (We note that for large amplitude motions, buckling dynamics can also occur with a component perpendicular to this cutting plane. Furthermore, nanoscale roughness of the wedge surface might break the translational symmetry of the wedge and can generate some additional dynamics in a perpendicular direction to the cutting plane.) For the two-dimensional case (Figure 1a), the strain tensor consists of three independent components, $\varepsilon_{x}, \varepsilon_{z}$, and $\varepsilon_{x z}$. In Figure 4, we only display the temporal and spatial change of $\varepsilon_{x}$ and $\varepsilon_{z}$ as the off-diagonal component in our case (and for this choice of coordinate system) is small compared to the diagonal one.

Although the thermal stress generated by laser excitation (eq 8 ) is isotropic, the boundary conditions of the slab geometry give rise to strongly anisotropic strain dynamics. Initially, the isotropic stress generates a motion in the $z$-direction where the structure has the smallest thickness, that is, tip of the wedge. This dynamics is comparable to the breathing motion observed in thin films where the period of breathing is given by the sound velocity and the thickness. ${ }^{13}$ However, in the case of a wedge, the thickness varies along the $x$-direction, and the breathing motions evolve to have a different period along the wedge. This feature generates a "traveling" strain wave along the $x$-direction with the amplitude of the motion being along the $z$-direction (transverse wave, Figure 4, left panel). The traveling wave generates an additional motion with an amplitude in the $x$-direction (Figure 4, right panel).

Due to the Poisson ration of the material, the breathing motion in the $z$-direction induces a stress in the $x$-direction. For thin-films, this stress is laterally homogeneous and hence does not generate any lateral motion. However, for a wedge the induced stress by the breathing motion is laterally inhomogeneous and thus generates a secondary, oscillatory motion in the $x$-direction. The lateral modulation of the strain is on the order of $100 \mathrm{~nm}$, which clearly demonstrates the need for nanoscale probes in order to visualize such dynamics; a parallel beam illumination would be blind to such modulations.

To better visualize these dynamics, we considered the strain components over a small probing region, as shown in Figure 4. The resulting temporal changes of the strain are displayed in Figure 5. It is evident that they oscillate with a well-defined

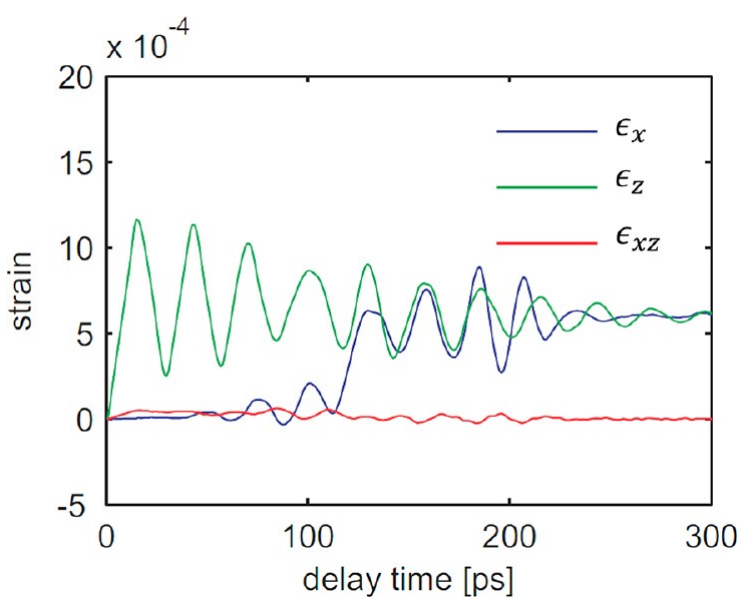

Figure 5. Strain temporal dynamics when the convergent electron beam probes at fixed position on the specimen. Shown are the temporal behavior of the $x$ - (blue curve), $z$ - (green curve), and $x z$ component (red curve) of the strain summed over the probed volume. Periodic oscillations at $30 \mathrm{ps}$ are evident. As importantly, the $z$ component depicts the typical damping (exponential) behavior of breathing modes, whereas the $x$-component of strain exhibits a delayed transit behavior with an envelope; the period is 30 ps with an onset near $100 \mathrm{ps}$, as observed experimentally. 
period of $30 \mathrm{ps}$, a value set by the thickness of the slab at the probed region and the speed of sound. Most importantly for our discussion, the strain dynamics in the $z$-direction begins following the ultrafast laser excitation, on a time scale which is only limited by the thermal stress buildup; it shows the typical (exponentially) damping behavior. However, the strain along the $x$-direction has a transient-like dynamics. It is initiated by the primary breathing motion and builds up gradually on a time scale of 100 ps as a secondary consecutive process should do. This temporal behavior closely resembles the experimentally observed shift of the Kikuchi band positions shown in Figure 2 .

Conclusion. Convergent, instead of parallel, beam ultrafast electron microscopy enables observations of structural dynamics that are unique to complex and heterogeneous structures, such as the one studied here (wedge slab shape). The transverse and longitudinal eigen modes of the structure are evident in Kikuchi diffraction. The simultaneous temporal and spatial probing of 4D UEM made it possible to produce movies of propagating elastic waves with (sub)nanometer and ultrafast time resolutions. Several disciplines concerned with defects and their propagations, ${ }^{14}$ strain-stress at the microscopic level, ${ }^{15}$ and even transport in biological cells ${ }^{16}$ may benefit from this convergent beam.

\section{AUTHOR INFORMATION}

\section{Corresponding Author}

*E-mail: zewail@caltech.edu.

\section{Notes}

The authors declare no competing financial interest.

\section{ACKNOWLEDGMENTS}

This work was supported by the National Science Foundation (DMR-0964886) and the Air Force Office of Scientific Research (FA9550-11-1-0055) in the Gordon and Betty Moore Center for Physical Biology at the California Institute of Technology.

\section{REFERENCES}

(1) Zewail, A. H.; Thomas, J. M. 4D Electron Microscopy; Imperial College Press: London, 2009.

(2) Yurtsever, A.; Zewail, A. H. Kikuchi ultrafast nanodiffraction in four-dimensional electron microscopy. Proc. Natl. Acad. Sci. U.S.A. 2011, 108, 3152-3156.

(3) Batson, P. E. Unlocking the time resolved nature of electron microscopy. Proc. Natl. Acad. Sci. U.S.A. 2011, 108, 3099-3100.

(4) Yurtsever, A.; Zewail, A. H. 4D Nanoscale Diffraction Observed by Convergent-Beam Ultrafast Electron Microscopy. Science 2009, 326, 708-712.

(5) Yurtsever, A.; van der Veen, R. M.; Zewail, A. H. Subparticle Ultrafast Spectrum Imaging in 4D Electron Microscopy. Science 2012, 335, 59-64.

(6) Cowley, J. M.; Spence, J. C. H. Convergent Beam Electron Microdiffraction from Smal Crystals. Ultramicroscopy 1981, 6, 359336.

(7) Wang, Z. L. Elastic and inelastic scattering in electron diffraction and imaging; Plenum Press: New York, 1995.

(8) Spence, J. C. H. High-resolution electron microscopy, 3rd ed.; Oxford University Press: Oxford, 2003.

(9) Morniroli, J. P. Large-angle convergent-beam electron diffraction (LACBED): Applications to crystal defects; Société Française des Microscopies: Paris, 2002.

(10) Harb, M.; Ernstorfer, R; Dartigalongue, T.; Hebeisen, C. T.; Jordan, R. E.; Miller, R. J. D. Carrier Relaxation and Lattice Heating Dynamics in Silicon Revealed by Femtosecond Electron Diffraction. J. Phys. Chem. B 2006, 110, 25308-25313.
(11) Sjodin, T.; Petek, H.; Dai, H.-L. Ultrafast Carrier Dynamics in Silicon: A Two-Color Transient Reflection Grating Study on a (111) Surface. Phys. Rev. Lett. 1998, 81, 5664-5667.

(12) Sjodin, T.; Li, C.-M.; Petek, H.; Dai, H.-L. Ultrafast transient grating scattering studies of carrier dynamics at a silicon surface. Chem. Phys. 2000, 251, 205-213.

(13) Barwick, B.; Park, H. S.; Kwon, O.-H.; Baskin, J. S.; Zewail, A. H. 4D Imaging of Transient Structures and Morphologies in Ultrafast Electron Microscopy. Science 2008, 322, 1227-1231.

(14) Robertson, I. M.; Schuh, C. A.; Vetrano, J. S.; Browning, N. D.; Field, D. P.; Jensen, D. J.; Miller, M. K.; Baker, I.; Dunand, D. C.; Dunin-Borkowski, R.; Kabius, B.; Kelly, T.; Lozano-Perez, S.; Misra, A.; Rohrer, G. S.; Rollett, A. D.; Taheri, M. L.; Thompson, G. B.; Uchic, M.; Wang, X.-L.; Was, G. Towards an integrated materials characterization toolbox. J. Mater. Res. 2011, 26, 1341-1383.

(15) Zhang, P.; Istratov, A. A.; Weber, E. R.; Kisielowski, C.; He, H.; Nelson, C.; Spence, J. C. H. Direct strain measurement in a $65 \mathrm{~nm}$ node strained silicon transistor by convergent-beam electron diffraction. Appl. Phys. Lett. 2006, 89, 161907-161903.

(16) Lippincott-Schwartz, J.; Smith, C. L. Insights into secretory and endocytic membrane traffic using green fluorescent protein chimeras. Curr. Opin. Neurobiol. 1997, 7, 631-639. 